\title{
IDENTIFICACIÓN DE DIFICULTADES DE LECTURA EN ESTUDIANTES DE PRIMERO Y TERCERO DE EDUCACIÓN PRIMARIA
}

\author{
Laura Gorjón Palos \\ Educadora Social \\ lauragp1992@hotmail.com \\ Ana Isabel Isidro de Pedro \\ Universidad de Salamanca \\ anyis@usal.es
}

Recepción Artículo: 22 enero 2020

Admisión Evaluación: 4 marzo 2020

Informe Evaluador 1: 1 abril2020

Informe Evaluador 2: 7 abril 2020

Aprobación Publicación: 20 abril 2020

\section{RESUMEN}

Este trabajo investiga las dificultades del aprendizaje en la lectura en menores que cursan Primero y Tercero de Educación Primaria. El propósito principal fue detectar dificultades de aprendizaje de lectura en Primaria con la finalidad de que el centro educativo realice una intervención preventiva. La muestra estuvo conformada por 109 estudiantes, con edades comprendidas entre los 6 y 10 años. Para ello, se realizó un estudio empírico en el que se aplicaron las pruebas siguientes: Prueba de Nivel de Lectura Global (TECLE), una prueba de lectura de palabras (PIPE), una prueba de ortografía (ORT) y dos pruebas para la evaluación de las representaciones metafonológicas (SIL y FON). Los resultados muestran que es posible detectar precozmente dificultades de lectura. A partir de dicha detección, los centros escolares pueden llevar a cabo una intervención temprana para que tales dificultades sean mitigadas o subsanadas.

Palabras clave: educación primaria; dificultades de aprendizaje de la lectura; atención temprana; detección precoz; comprensión lectora

\section{ABSTRACT \\ Pay attention to diversity: Identification of reading difficulties in schoolchildren of first and third grades of primary education. This work investigates the learning difficulties in reading in schoolchildren who are in the First and the Third grades of Primary Education. The main purpose was to detect reading learning difficulties in Primary Education in order to that the educational center performs a preventive intervention. The sample consisted of 109 schoolchildren aged between 6 and 10 years. For that, an empirical study was carried out in which the following tests were applied: a Global Reading Level Test (TECLE), a word reading test (PIPE), a spelling test (ORT) and two tests for evaluation of the metaphonological representations (SIL}




\section{IDENTIFICACIÓN DE DIFICULTADES DE LECTURA EN ESTUDIANTES DE PRIMERO Y}

TERCERO DE EDUCACIÓN PRIMARIA

and FON). The results show that it is possible to detect reading difficulties early. From this detection, schools can perform an early intervention so that such difficulties are mitigated or remedied.

Keywords: primary education; reading learning difficulties; early attention; early detection; comprehensive reading

\section{INTRODUCCIÓN}

El aprendizaje y la enseñanza de la lectura constituyen un pilar fundamental del ser humano. Este aprendizaje es definitivo, especialmente en las primeras etapas educativas (National Early Literancy Panel, 2008) y esencial para el desarrollo escolar, social y laboral. Este artículo analiza este aprendizaje desde el punto de vista de la prevención o detección precoz de dificultades en el mismo. El trabajo aquí presentado muestra un estudio para evidenciar si las pruebas de evaluación planteadas en dicho proyecto de investigación son válidas para la detección precoz de dificultades de aprendizaje de la lectura, objetivo que forma parte del II Plan de Atención a la Diversidad que actualmente está desarrollando la Junta de Castilla y León.

Este trabajo se fundamenta en el Modelo Simple de Lectura (Simple View of Reading), propuesto por Hoover y Gough (1990), que es un modelo de lectura flexible, puede ser la base sobre la que se asientan las decisiones que se toman para los programas de enseñanza y proporciona una manera sencilla de comprender las habilidades implicadas en el aprendizaje de la lectura; con lo cual, esto nos da la posibilidad de investigar dificultades en dicho aprendizaje.

El Modelo Simple de Lectura plantea que la comprensión lectora es el resultado de la interacción entre la descodificación y la comprensión del lenguaje, que puede ser evaluada mediante pruebas de comprensión oral con un contenido similar al empleado para evaluar la comprensión lectora (Zeballos, Arcos y Ripoll, 2017).

Este trabajo se centra en la parte de descodificación. Teniendo en cuenta este modelo teórico, para ser un buen lector hay que tener dos habilidades notorias: habilidad para reconocer palabras escritas y habilidad de comprensión oral. Ambas son componentes relevantes de la lectura. Reconocer la palabra escrita significa que el lector debe aprender las reglas de correspondencia entre grafemas y los fonemas, es decir, tiene que entender cómo se relacionan los símbolos gráficos con los sonidos (Defior y Serrano, 2011). Es importante, señalar que la decodificación es un proceso básico donde se asigna un sonido a un estímulo visual, mientras que identificar palabras escritas consiste en añadir significado a esos sonidos y estímulos visuales. Entonces, identificar una palabra es acceder a la información fonológica, semántica y ortográfica que se tiene sobre la misma. Al practicar esa habilidad, el reconocimiento de las palabras escritas se hace más fluido y se convierte en un proceso automático, donde no se gastan muchos recursos de atención y memoria. Por ello, cuando se utilizan pocos recursos hacia esta operación, mayor número de recursos se utilizan para actividades más complejas tales como la comprensión.

Para analizar estos procesos nos centraremos en el modelo de doble ruta, según el cual existen dos rutas 0 procedimientos para procesar las palabras. Por un lado, está la ruta directa o léxica, la cual nos lleva de manera directa a la forma ortográfica de las palabras, es decir, se asocian las palabras directamente con su significado; esta es la ruta que nos permite leer palabras frecuentes. Pero, por otro lado, está la ruta indirecta o subléxica en la que el lector dispone de reglas para convertir los grafemas en sonidos que le permiten recodificar fonológicamente la palabra escrita, es decir, va convirtiendo cada letra en un sonido correspondiente hasta que pronuncia la palabra entera (Ripoll y Aguado, 2015). Ambas vías están íntimamente relacionadas en la lectura hábil, como parte de un mismo proceso. Utilizar un tipo de vía u otra depende de los conocimientos y experiencias lingüísticas que el lector tiene sobre las palabras. Dichos conocimientos trabajan simultáneamente para identificar de manera rápida las palabras, siendo mayor o menor la aportación de cada uno de esos conocimientos dependiendo del tipo de palabra a leer (frecuente o no frecuente) y de las habilidades del lector (Defior, 2014).

Un buen lector se caracteriza por el uso eficaz y automático de ambas vías, es decir, lee con precisión y con automaticidad. Ambas capacidades componen lo que se denomina fluidez lectora, una de las cinco habilidades que señala National Reading Panel (2000) en las que se basa el éxito del lector. 
El National Reading Panel habla de cinco habilidades denominadas las cinco grandes de la lectura, donde se pretende enseñar con éxito a los niños a leer e impedir dificultades que aparecen en diversas ocasiones. Estas cinco habilidades son: conciencia fonológica, principio alfabético, fluidez, vocabulario y comprensión de textos (Armbruster, Lehr, Osborn y Adler, 2009). Estas capacidades son algunas de las evaluadas en la parte empírica de este trabajo.

En primer lugar, la conciencia fonológica es la capacidad de ser consciente de la existencia de los fonemas dentro de la lengua y ser capaz de manipularlos. Antes de que un niño aprenda a leer una letra, necesita ser consciente de cómo funcionan los sonidos de cada palabra. En este trabajo se emplean dos tareas para evaluar esta capacidad: la Prueba de detección de sílabas y otra tarea de fonemas, de la Batería PEALE (Domínguez et al., 2014).

En segundo lugar, el principio alfabético es la comprensión de que existe una relación previsible entre fonemas y grafemas. Es decir, los niños que no puedan oír y trabajar con los fonemas de las palabras habladas tendrán problemas para aprender a relacionar estos fonemas con los grafemas cuando vean esas palabras escritas. En definitiva, el acomplamiento entre grafemas y fonemas facilitara al estudiante alcanzar reconocimiento de palabras, basado en un conocimiento elemental relacionado con la ortografía de la lengua escrita. En este trabajo se emplea una prueba de identificación de palabras escritas (PIPE) (Domínguez et al., 2014) para evaluar esta capacidad y la siguiente, esto es, la fluidez lectora.

En tercer lugar, la fluidez es la capacidad de leer con precisión y rapidez y expresar palabras poniendo énfasis conveniente. Cuando los niños saben leer con soltura reconocen las palabras de forma automática y agrupan de manera rápida las palabras y así consiguen el significado de lo que leen. Sin embargo, aquellos lectores que no han adquirido esta habilidad leen más lentamente y de manera laboriosa.

La fluidez es adquirida de manera gradual, durante un tiempo considerable practicando de manera continua. Pero, por otro lado, dentro de la fluidez se encuentra la automaticidad y precisión, puesto que en diversas ocasiones se usan de manera similar y no son lo mismo. El primer constructo se refiere a reconocer la palabra rápidamente y sin esfuerzo. La precisión es la capacidad de leer sin cometer errores (Armbruster et al., 2014). Para analizar esta capacidad, en este trabajo se emplea, además de la prueba PIPE, la Prueba TECLE.

En cuarto lugar, el vocabulario referido a las palabras que debemos conocer para comunicarnos de manera efectiva. Existen dos tipos de vocabulario: el primero se denomina vocabulario oral y se refiere a las palabras que utilizamos al hablar o reconocer cuando se escucha. El segundo es el Ilamado vocabulario de lectura, que consiste en esas palabras que reconocemos. El vocabulario juega un papel muy importante en el aprendizaje de la lectura y comprensión lectora. Los alumnos aprenden vocabulario de manera indirecta, es decir, en distintos contextos, cuando oyen y ven palabras usadas en diferentes momentos, en conversaciones con adultos, a través de lo que leen o también aprenden vocabulario de manera directa, cuando están explícitamente aprendiendo palabras individuales (Armbruster et al., 2014).

Diversas investigaciones realizadas sobre la enseñanza de vocabulario revelan que la mayor parte del vocabulario se aprende de manera indirecta; sin embargo el vocabulario debería ser enseñado directamente, puesto que de esta manera, los estudiantes aprenderían conceptos complejos. En definitiva, cuanto mayor sea el número de palabras que conocen, mayor significado encontraran a la hora de leer (Goswami, 2011).

Finalmente, la comprensión significa que los buenos lectores pretenden y persiguen encontrar sentido en todo aquello que leen empleando sus conocimientos y experiencias para dar sentido al texto y sacar el mayor provecho de ella. Además, en la lectura pueden ajustar la velocidad teniendo en cuenta la dificultad del texto.

Teniendo en cuenta estas cinco habilidades, muchos trabajos señalan que es ineludible implementar programas preventivos e identificaciones tempranas, a la par que se realizan evaluaciones a los alumnos para detectar posibles dificultades y, a partir de ahí, apoyar y mejorar esos déficits. La identificación temprana es necesaria puesto que la prevención e intervención precoz constituyen las medidas educativas más eficientes para paliar la aparición de dificultades de aprendizaje de la lectura y facilitar el proceso de alfabetización (Infante, Coloma y Himmel, 2012). 


\section{IDENTIFICACIÓN DE DIFICULTADES DE LECTURA EN ESTUDIANTES DE PRIMERO Y TERCERO DE EDUCACIÓN PRIMARIA}

Concordando con ello, Cuevas (2005) afirma que es importante que se produzca una atención temprana porque las dificultades de aprendizaje se presentan en la infancia con más frecuencia de lo que se piensa, pero en muchas ocasiones se diagnostican tarde o no se detectan. Acorde con esto, Jiménez, Guzmán y Rodríguez (2009) señalan también la importancia de la detección temprana, lo que implicaría la detección de los niños de alto riesgo de presentar estas dificultades.

\section{OBJETIVO}

Teniendo en cuenta lo anterior, el objetivo fundamental de este trabajo es detectar dificultades de aprendizaje de lectura en alumnado de Primero y Tercero de Educación Primaria con la finalidad de que el centro educativo pueda implementar una intervención preventiva.

\section{METODOLOGÍA}

En esta investigación participaron 109 estudiantes de Primero y Tercero de Educación Primaria de dos centros concertados de zona rural y urbana de la provincia de Salamanca. El grupo tenía edades comprendidas entre 6 y 9 años. De los 109 niños, 47 eran de Primero de Primaria y 63 de Tercero de Primaria.

\section{Diseño}

El diseño utilizado fue un diseño trasversal de medidas tomadas en un mismo momento en los diversos grupos de alumnos.

\section{Instrumentos}

Para la realización de este trabajo se utilizaron 5 pruebas que permitieron realizar una detección precoz de posibles dificultades de aprendizaje de la lectura: Una prueba de Nivel de Lectura Global (TECLE), una prueba de lectura de palabras (PIPE), una prueba de ortografía (ORT) y dos pruebas para la evaluación de las representaciones metafonológicas (SIL y FON).

- Test de Eficiencia Lectora (TECLE) (Marín y Carrillo, 1999). Esta prueba proporciona un nivel de lectura global, se compone de 64 frases incompletas a las que les falta una palabra que se ha de elegir entre cuatro opciones de respuesta. A medida que avanza la prueba va aumentando su dificultad ortográfica, sintáctica y su longitud. Los participantes disponen de cinco minutos para completar el mayor número de frases. La corrección de la prueba es el número de aciertos menos el número de errores dividido por tres, para eliminar el factor azar. Por ejemplo: "Su hermano estudia en la... universidad, unidersidad, unipersonal, universilad. La fiabilidad de la prueba evaluada mediante el alpha de Cronbach y la prueba de mitades alcanzó valores elevados. ( $\alpha=.979$ y r=.994).

- Prueba de Decisión Ortografía (D0) (Domínguez, et al., 2013). Esta prueba evalúa la calidad de las representaciones ortográficas de los participantes. Se proporcionan dos palabras, la correcta y su pseudomófono. No se dispone de tiempo para completar la prueba. Un ejemplo de los que deben responder sería: gaviota/ gabiota. La fiabilidad de la prueba evaluada mediante el alpha de Cronbach y la prueba de mitades alcanzó valores elevados $(\alpha=.911$ y $r=.915)$.

- Pruebas de Metafonología (Domínguez, et al., 2013). Esta prueba está formada por dos subpruebas que evalúan no solo la presencia de representaciones fonológicas sino la capacidad para su manipulación. Están conformadas por 90 dibujos que representan objetos de la vida cotidiana y se dispone de tres minutos para la realización de cada subprueba.

- Subprueba de Sílabas (SIL): Ios alumnos deben ir señalando el número de sílabas de cada uno de los dibujos, rodeando en círculo los números (1, 2, 3, 4 o 5) ubicados debajo de cada imagen. Un ejemplo de esta prueba sería: la imagen de un cocodrilo, cuya palabra tiene cuatro sílabas, entonces deberán rodear el número 4. La fiabilidad de la prueba evaluada mediante el alpha de Cronbach y la prueba de mitades alcanzó valores elevados $(\alpha=.970$ y $r=.990)$.

- Subprueba de Fonemas (FON): Ios estudiantes deben contar los fonemas que tiene el nombre de cada ima- 
gen y marcar el número que corresponda: $(2,3,4,506)$. Por ejemplo: en la imagen de un camión deberán rodear número 6. La fiabilidad de la prueba evaluada mediante el alpha de Cronbach y la prueba de mitades alcanzó valores elevados ( $\alpha=.962$ y $r=.985)$.

- Prueba de Identificación de Palabras Escritas (PIPE) (Domínguez et al. 2014). Esta prueba solo fue completada por los alumnos de Primero de Primaria. Se proporciona una imagen y tres palabras, el estudiante debe elegir qué palabra representa el dibujo dado. Por ejemplo: en la imagen de una escalera, tiene opción de señalar: escalada, escalera, escopeta. Esta prueba consta de 60 ítems y disponen de tres minutos para señalar el mayor número de palabras. La fiabilidad de la prueba evaluada mediante el alpha de Cronbach y la prueba de mitades alcanzó valores elevados ( $\alpha=.956$ y $r=.993)$.

\section{Procedimiento}

La recogida de datos fue realizada de manera colectiva en cada una de las clases, en tres sesiones: en la primera sesión TECLE, ORT y SIL, en la segunda sesión PEES, FON y STX y en la tercera VOC, TON y, en el caso de los alumnos de Primero de Educación Primaria, PIPE.

Todas las pruebas fueron presentadas y explicadas verbalmente una a una, realizando varios ejemplos colectivos con el fin de comprobar que todos los niños habían entendido las instrucciones.

\section{RESULTADOS}

Como se ha dicho, el objetivo de este trabajo fue detectar dificultades de la lectura en estudiantes de Primero y Tercero de Educación Primaria. Para ello, se analizaron las Puntuaciones Directas Medias obtenidas por los participantes en cada una de las pruebas.

Las Figuras 1 y 2 muestran las puntuaciones medias obtenidas por los participantes, en las pruebas utilizadas para este estudio.

Figura 1. Media Puntuación Directa 19 Primaria

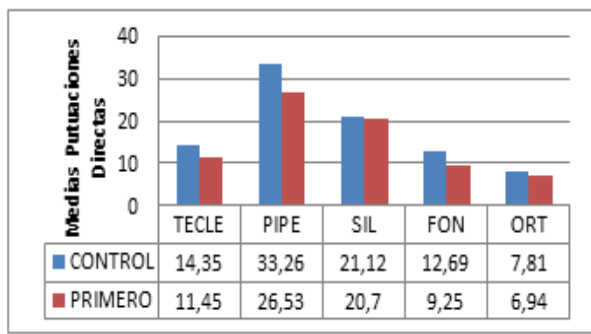

Figura 2. Media Puntuación Directa 3ํ Primaria

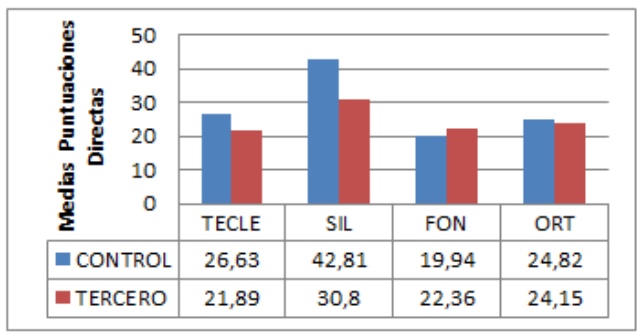

Se aprecia que las puntuaciones de los grupos experimentales son inferiores a las obtenidas por el grupo control en todas las pruebas. Los resultados obtenidos por los estudiantes de Primero de Primaria indican que la mayor diferencia se encuentra en la prueba de identificación de palabras escritas -donde la diferencia entre el grupo experimental y el control es de siete puntos- y la menor estaría en la prueba ortográfica -donde existe una diferencia de un punto-.

Los resultados de Tercero de Primaria ponen de manifiesto la mayor diferencia en la prueba de sílabas donde el grupo control supera en 12 puntos al grupo experimental- y la menor en la prueba de Decisión Ortográfica -cuya diferencia es inferior a un punto-. Sin embargo, en la prueba de fonemas el grupo experimental tiene una puntuación de dos puntos por encima del grupo control.

Para un análisis más detallado de las variables implicadas en este estudio, la Figura 3 muestra las puntuaciones obtenidas en la prueba TECLE por los estudiantes de Primero de Primaria. 
Figura 3. Puntuaciones obtenidas en la prueba de Nivel lector en estudiantes de Primero de Primaria

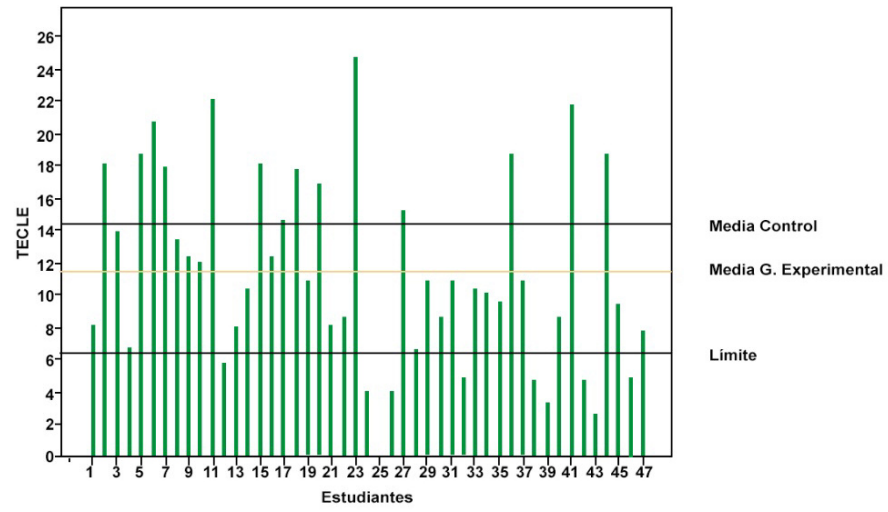

Se aprecian tres líneas, la Media obtenida por el grupo control, la media obtenida por el grupo experimental y, finalmente, la puntuación límite: esta puntuación ha sido calculada a partir de la media obtenida por el grupo control, restando a este valor 1.5 desviaciones típicas. Esta puntuación indica el valor por debajo del cual se puede considerar la detección precoz de dificultades de aprendizaje de la lectura.

Observamos que la media del grupo experimental es inferior a la del grupo control. La mayor parte de los estudiantes que conforman el grupo experimental se sitúan por debajo de la media control e, incluso, de la media del grupo experimental. Los estudiantes número 24,26,32,38,39,42,43 y 46 se encuentran por debajo de la puntuación límite, con lo que sería necesario analizar con mayor profundidad el resto de variables que intervienen en la comprensión lectora.

Figura 4. Puntuaciones obtenidas en la prueba PIPE en estudiantes de Primero de Primaria

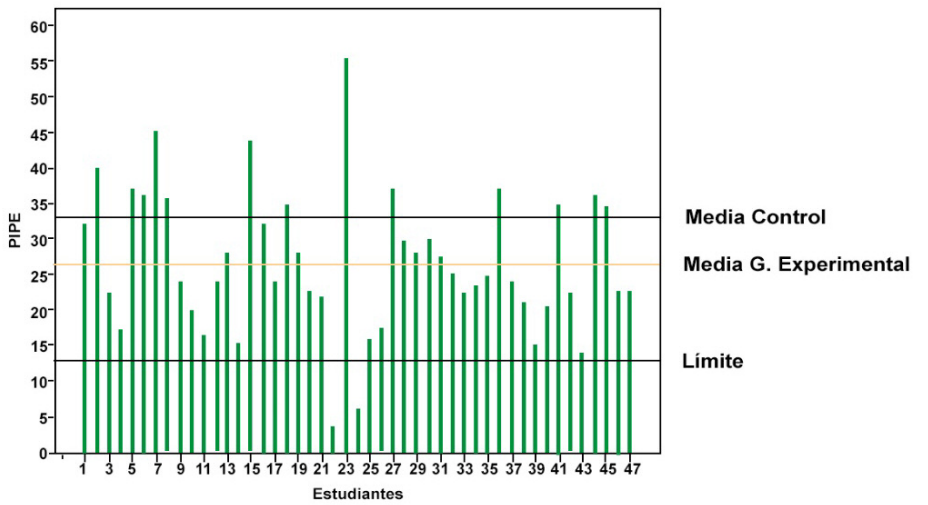

En la prueba PIPE (ver Figura 4), la media del grupo experimental es inferior a la media del grupo control. La puntuación límite se establece en 12.71. Los estudiantes número 22 y 24 se encuentran por debajo de esta puntuación, por lo que sería prioritario intervenir con esos estudiantes para que las dificultades no se agravaran con el tiempo. Como puede observarse la media de grupo experimental está por debajo de la media control y se 
aprecia que existen diversos estudiantes con puntuaciones por debajo de la media de su grupo. Por el contrario, los estudiantes 2,7, 18 y 23 se encuentran por encima de la media del grupo control.

Figura 5. Puntuaciones obtenidas en la prueba de Sílabas por estudiantes de Primero de Primaria.

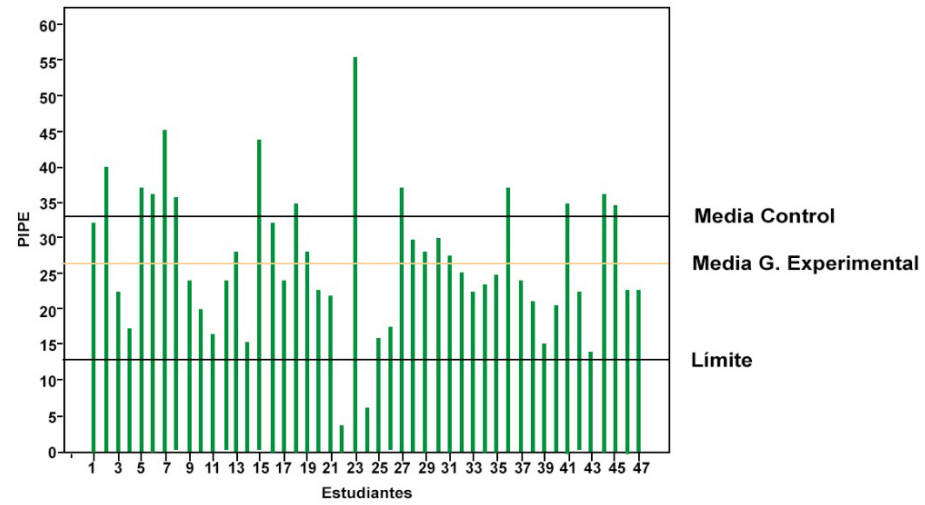

En la prueba de Sílabas (ver Figura 5), la media del grupo experimental es similar a la media del grupo control. En este caso, la puntuación límite es 2.71 y solamente el estudiante número 19 se encuentra por debajo de esta puntuación, lo cual indica las dificultades que presenta con el manejo de sílabas dentro de palabras. También se aprecia que los estudiantes 1,2,7 y 18 están muy por encima de la media control y de la media del grupo experimental.

Figura 6. Puntuaciones obtenidas en la prueba de Fonemas en estudiantes de Primero de Primaria.

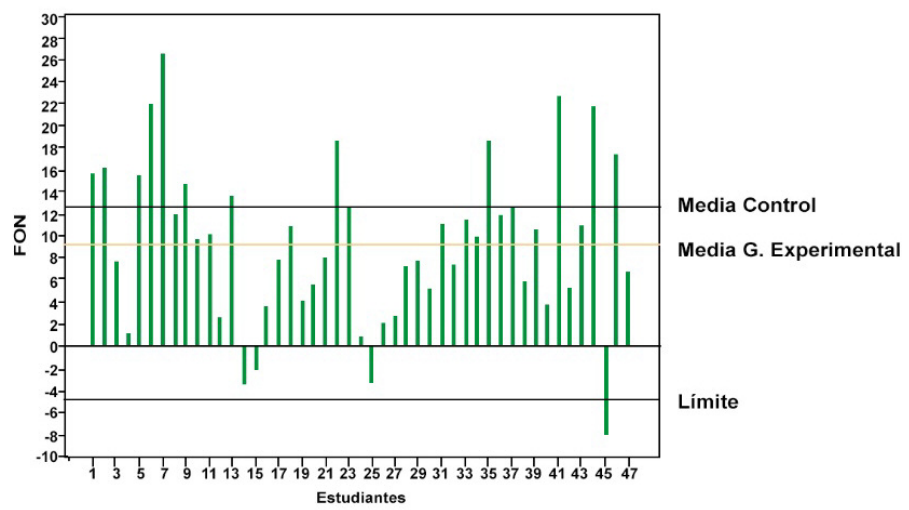

Los resultados obtenidos en la prueba de Fonemas (ver Figura 6) muestran que el límite es de -4.78 , siendo el estudiante 45 el único situado por debajo de esta puntuación. No obstane, hay otros estudiantes (14, 15 y 25$)$ que se acercan de manera notable a dicho límite. Asimismo, se observa que los estudiantes 7, 22, 41 y 46 superan de manera significativa la media del grupo experimental y la media control. 


\section{IDENTIFICACIÓN DE DIFICULTADES DE LECTURA EN ESTUDIANTES DE PRIMERO Y TERCERO DE EDUCACIÓN PRIMARIA}

Figura 7. Puntuaciones obtenidas en la prueba de Ortografía en estudiantes de Primero de Primaria

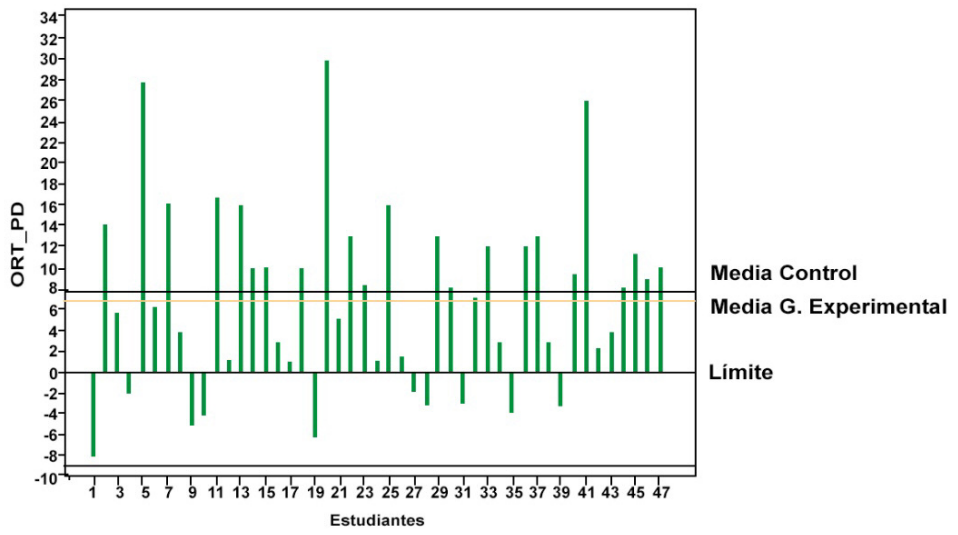

En la Figura 7 aparecen las puntuaciones directas obtenidas por los estudiantes de Primero de Primaria en la prueba de Ortografía. Observamos que la media del grupo control es muy similar a la media del grupo experimental, pero puede considerarse que hay gran número de estudiantes que no llegan a la media del grupo experimental. Sin embargo, otros valores exceden la media del grupo control (estudiantes 2, 5, 20, 25 y 41).

Por otro lado, la puntuación límite es de -9.00, pero hay alumnos (1, 9,19, 31 y 39) que lo superan notablemente.

A continuación, se procede al análisis más detallado de las puntuaciones medias obtenidas por los estudiantes de Tercero de Primaria.

Figura 8. Puntuaciones obtenidas en la prueba de Nivel lector en estudiantes de Tercero de Primaria

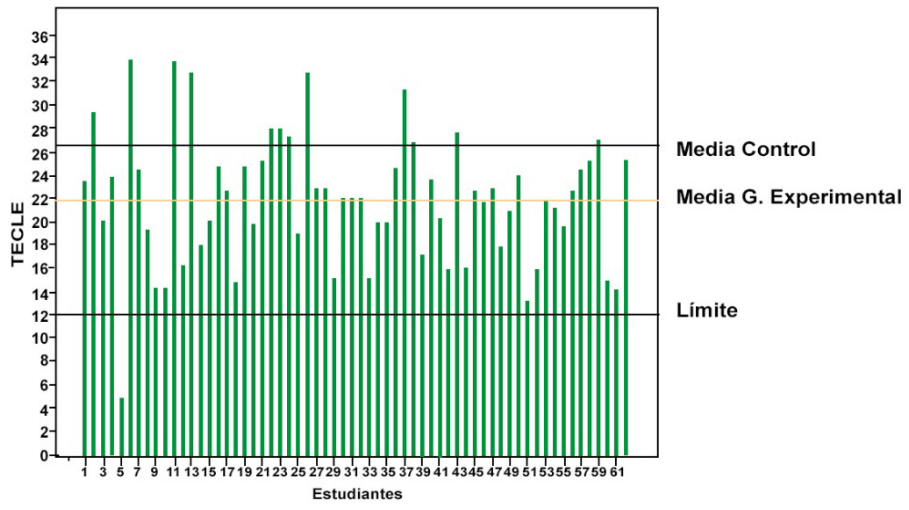

Como puede observarse, la Figura 8 representa las puntuaciones obtenidas en la prueba de Nivel lector en estudiantes de Tercero de Primaria. La media del grupo control es mayor a la del grupo experimental. Varios estudiantes del grupo experimental $(6,11,13$ y 26$)$ se sitúan muy por encima de la media control. Pero existen otros estudiantes que se encuentran por debajo de la media control, con lo cual sería relevante analizar las variables que intervienen en la comprensión lectora. Además, observamos que el estudiante 5 se encuentra por debajo de esta puntuación límite, puesto que dicha puntuación se establece en 11.92, con lo cual habría que realizar una evaluación más exhaustiva. 
Figura 9. Puntuaciones obtenidas en la prueba de Sílabas por estudiantes de Tercero de Primaria.

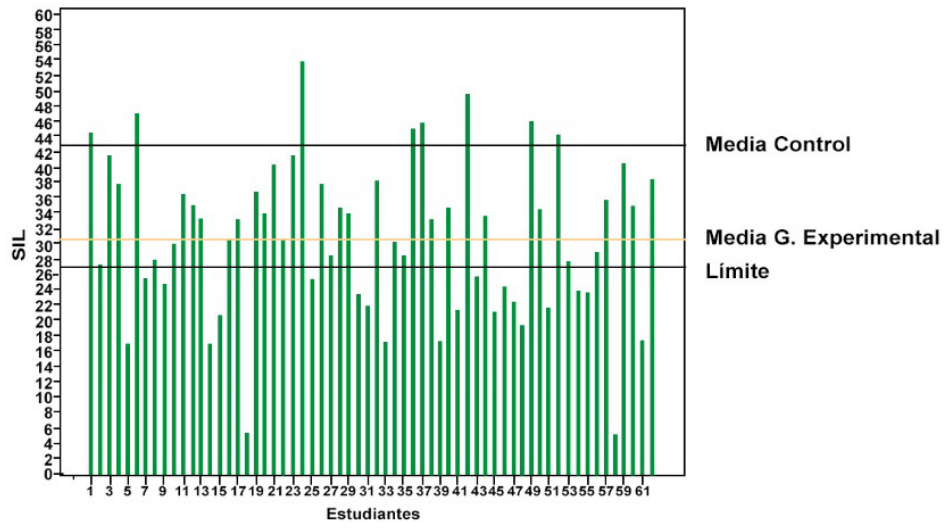

La Figura 9 representa las puntuaciones en la prueba de Sílabas en estudiantes que cursan Tercero de Primaria. Se aprecia que la puntuación límite se acerca en gran medida a la puntuación media experimental, con lo que el número de estudiantes que se encuentran por debajo de esta puntuación es bastante importante, pero destacan los estudiantes 18 y 58 cuyas diferencias son aún mayores.

La media del grupo experimental es inferior a la del grupo control. La mayor parte de los estudiantes que conforman el grupo experimental se sitúan por debajo de esta media, aunque cabe destacar que los estudiantes 30 y 42 destacan en mayor medida, puesto que tienen puntuaciones mayores que la media del grupo control.

Figura 10. Puntuaciones obtenidas en la prueba de Fonemas en estudiantes de Tercero de Primaria

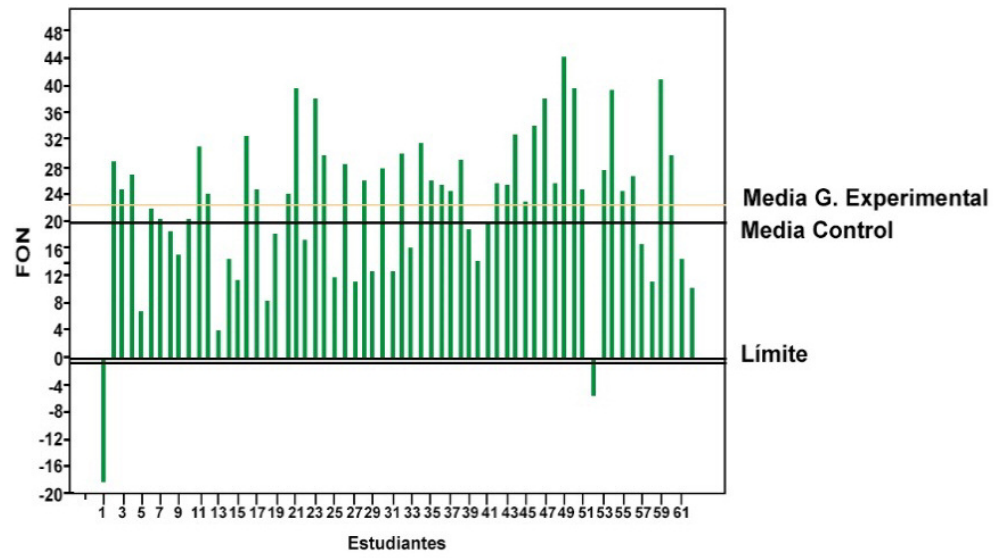

En la Figura 10, se muestra el nivel fonémico en Tercero de Primaria. Se aprecia que la media del grupo experimental es superior a la del grupo control, aunque apenas existen diferencias entre ambos. La puntuación límite alcanzó el valor de -.68, dejando por debajo de la misma a los estudiantes 1 y 52. 


\section{IDENTIFICACIÓN DE DIFICULTADES DE LECTURA EN ESTUDIANTES DE PRIMERO Y}

TERCERO DE EDUCACIÓN PRIMARIA

Figura 11. Puntuaciones obtenidas en la prueba de Ortografía en estudiantes de Tercero de Primaria

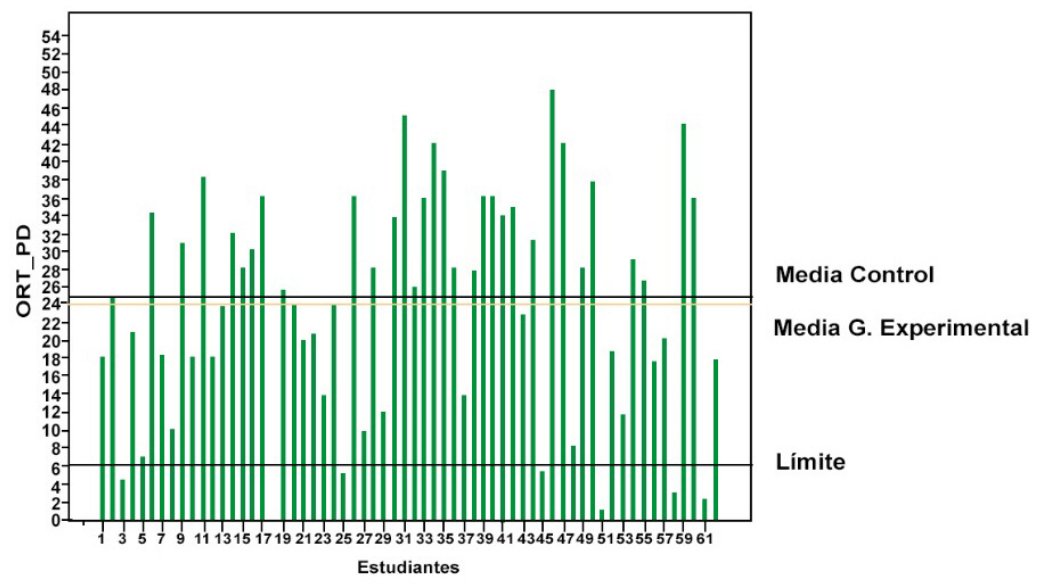

En la prueba de Ortografía (ver Figura 11), la media del grupo experimental y la media del grupo control son similares y no existen apenas diferencias apreciables. La puntuación límite se establece en 6.45. Los estudiantes $3,18,25,45,51,58$ y 61 se encuentran por debajo de esta puntuación. Sin embargo, gran parte de los estudiantes del grupo experimental se encuentran por debajo de su puntuación media. Y varios (31, 46 y 59) están muy por encima de la media del grupo control.

\section{CONCLUSIONES}

La Asociación Internacional de Dislexia refiere que entre el 15 y el 20\% de la población tiene dificultades de aprendizaje basada en el lenguaje. Lyon, Shaywitz y Shaywitz (2003) concuerdan también con esta estimación. Además, entre un 70 y un 80\% de los estudiantes que reciben educación especial muestran dificultades en la lectura y esta dificultad es la causa más frecuente de problemas en la escritura y las matemáticas (Asociación Internacional de Dislexia, 2003). La prevalencia del trastorno de lectura en niños en edad escolar es de un 4\% y no existen investigaciones a nivel nacional que reporten datos de prevalencia de dislexia (De los Reyes Aragón, Lewis y Peña, 2008).

En el estudio aquí plasmado, las mayores deficiencias se encontraron en las pruebas de precisión, y velocidad, pero no en conciencia fonológica. Se podría afirmar, que en esta investigación los estudiantes de Tercero de Primaria no presentan dificultades en la ruta fonológica, o segunda vía, a través de la cual se transforman los signos gráficos en sonidos y se llega al significado (Kolb y Whisaw, 1999). En esta vía, el lector reconoce los grafemas y la relación con los fonemas que componen, la palabra hablada. De este modo, los signos gráficos son convertidos en sonidos, mediante el sistema de conversión grafema-fonema y es a través de los sonidos como se accede al significado de las palabras. Siguiendo a Kolb y Whisaw (1999), esta vía se utiliza por los lectores en su fase inicial y a medida que perfeccionan la habilidad se vuelve más importante el procedimiento a partir de la vía léxica o visual; razón por la que los resultados obtenidos deberían tenerse en cuenta para la ejecución de los procesos de intervención escolar de los estudiantes con dificultades.

Asimismo, las pruebas Nivel de lectura global (TECLE), prueba de Lectura de palabras (PIPE), prueba de Ortografía (ORT) y pruebas para evaluar las representaciones metafonológicas (SIL Y FON) reflejaron niveles más bajos en todas ellas, pero no en los estudiantes de Tercero de Primaria que tenían mayores niveles en la prueba de fonemas. Esto implica una dificultad en la fluidez lectora, donde se tiene en cuenta la automaticidad y la precisión. 
En coherencia con lo anterior, algunos de los estudiantes presentaban dificultades en la manipulación de fonemas, realizaban omisiones o su pronunciación era errónea en algunas palabras de composición compleja.

Obviamente, Ios resultados de este trabajo se limitan a la muestra analizada y, por ende, no representan la prevalencia poblacional; por ello, son necesarios futuros estudios para seguir avanzando en la prevalencia de estas dificultades. Aun con las limitaciones y dificultades surgidas en este trabajo, es posible proponer que estos resultados son dignos de tener en cuenta para proceder a detectar dificultades en el aprendizaje de la lectura.

El análisis desarrollado a lo largo de este trabajo lleva a concluir que las dificultades ligadas a la lectura pueden, en múltiples ocasiones, pasar desapercibidas a los docentes de los centros escolares, conllevando que el alumno acarree este tipo de dificultades a lo largo de su vida educativa, personal y familiar. Por ello, es prioritario que el profesorado conozca tales dificultades, sus causas, las consecuencias que derivan de las mismas y, por supuesto, la necesidad de actuar tempranamente.

\section{REFERENCIAS BIBLIOGRÁFICAS}

Armbruster, B.B., Lehr, F., Osborn, J. y Adler, C.R. (2009). Put reading first: The research building blocks of reading instruction: Kindergarten through grade 3. National Institute for Literacy.

Cuevas, P.G. (2005). Atención temprana: prevención, detección e intervención en el desarrollo (0-6 años) y sus alteraciones. Editorial Complutense.

Defior, S. (2014). Procesos implicados en el reconocimiento de las palabras escritas. Processes involved in the recognition of written words. Aula, 20, 25.

Defior, S. y Serrano, F. (2011). Procesos fonológicos explícitos e implícitos, lectura y dislexia. Revista Neuropsicología, Neuropsiquiatría y Neurociencias, 11 (1), 79-94.

Domínguez, A.B., Alegría, J., Carrillo, M. y Soriano, J. (2013). PEALE. Pruebas de Evaluación Analítica de Lengua Escrita. Universidad de Salamanca. Número de asiento registral: 00/2013/4067.

Domínguez et al. (2014). Elaboración de un programa de enseñanza de habilidades morfosintácticas para estudiantes sordos: efectos sobre las estrategias de lectura de frases. Investigación financiada por el MICINN (proyecto EDU2014-52739).

De los Reyes, A.C. Lewis, H.S. y Peña, O.M. (2008). Estudio de prevalencia de dificultades de lectura en niños escolarizados de 7 años de Barranquilla (Colombia). Psicología desde el Caribe, 22, 37-49.

Goswami, U. (2011). A temporal sampling framework for developmental dyslexia. Trends Incognitive Sciences, $15(1), 3-10$.

Hoover, W.A. y Gough, P.B. (1990). The simple view of reading. Reading and Writing: An Interdisciplinayr Journal, 2, 127-160.

Infante, M., Coloma, C.J. y Himmel, E. (2012). Comprensión lectora, comprensión oral y decodificación en escolares de 2 y 4ํㅡásico de escuelas municipales. Estudios Pedagógicos, 38(1), 149-160.

Jiménez, J.E., Guzmán, R. y Rodríguez, C. (2009). Prevalencia de las dificultades específicas de aprendizaje: la dislexia en español. Anales de Psicología, 25(1), 78-85.

Kolb, B. y Whishaw, I.Q. (1999). Fundamentals of human Neuropsychology. Language. United States of America: W. H. Freeman and Company Worth Publishers.

Lyon, R.G., Shaywitz, S.E. y Shaywitz, B.A. (2003). Defining dyslexia, comorbidity, teacher's knowledge of language and reading. Annals of Dyslexia, 53,1-14.

Marín, J. y Carrillo, M.S. (1999). Test Colectivo de Eficacia Lectora (TECLE). Universidad de Murcia. Disponible en A. Cuadro, D. Costa, D. Trías, y P. Ponce de León (2009). Evaluación del nivel lector. Manual técnico del test de Eficacia Lectora (TECLE).

National Early Literacy Panel (2008). Developing early literacy: Report of the National Early Literacy Panel. Washington, DC: National Institute for Literacy.

Ripoll, J.C. y Aguado, G. (2015).Enseñar a leer. Cómo hacer lectores competentes. En J.C. Ripoll y G. Aguado (Ed.), Modelo de lectura (pp.15-38). Madrid: EOS. 
IDENTIFICACIÓN DE DIFICULTADES DE LECTURA EN ESTUDIANTES DE PRIMERO Y

TERCERO DE EDUCACIÓN PRIMARIA

Zevallos, D.S., Arcos, N.P. y Ripoll, J.C. (2017). La concepción simple de la lectura en alumnos de 4º de primaria de una escuela fiscal de Quito. Alteridad. Revista de Educación, 1, 115-122. 